\title{
Periodically refreshed multiply exposed photorefractive holograms
}

\author{
David Brady, Ken Hsu, and Demetri Psaltis \\ Department of Electrical Engineering, California Institute of Technology, Pasadena, California 91125
}

Received June 12, 1989; accepted April 19, 1990

\begin{abstract}
We describe a method for increasing the diffraction efficiency of multiply exposed photorefractive holograms by periodic copying. The method is experimentally demonstrated with photorefractive and thermoplastic recording media.
\end{abstract}

Photorefractive holograms are electro-optic index perturbations formed by the redistribution of photogenerated charge among local trapping sites. The charge pattern associated with a hologram is driven by its own space-charge field to decay exponentially with a time constant characterized by the dielectric relaxation time. In the dark, this time may be days or months. For applications in adaptive holography and holographic information storage, multiple exposures are used to form photorefractive holograms. Since the relaxation time decreases substantially during exposure, recording an uncorrelated hologram causes the partial erasure of previously recorded holograms. An exposure schedule by which an arbitrary number of holograms of equal diffraction efficiency may be recorded is described in Ref. 1 . Slightly different schedules directed at the same goal are described in Refs. 2 and 3. Unfortunately, the recording of multiple holograms according to this exposure schedule results in a diffraction efficiency for each hologram proportional to $M^{-2}$, where $M$ is the number of holograms recorded. This decrease in the diffraction efficiency limits the number of exposures to at most several hundred.

We describe a system in which part of the decrease in the diffraction efficiency of a multiply exposed hologram is recovered by periodic copying between two holographic media. Palais and Wise $\mathrm{W}^{4}$ demonstrated that copying a weak hologram onto a second medium, using large modulation depth, dramatically increases the diffraction efficiency of the second hologram. Johnson et al..$^{5}$ applied this technique to improve the diffraction efficiency of multiply exposed silver halide films. Here we demonstrate that periodic copying between two dynamic media improves the diffraction efficiency by a factor of $M$. A related result that we derive in this Letter is that periodic copying between two holograms results in a stable diffraction efficiency when an indefinitely long sequence of exposures is performed. Long sequences of exposures are used in adaptive holographic systems, such as optical perceptrons. ${ }^{6}$ Cross-modulation gratings and Bragg degeneracy problems have been considered ${ }^{7}$ for holographic associative memories, and we will not discuss these issues specifically for the periodic copying system considered here.

The architecture of the system is shown in Fig. 1. A series of holograms between a reference plane wave and a set of signal beams is recorded in a strontium barium niobate (SBN):Ce crystal. Shutters S4 and S5 are closed during this operation. We use plane waves as signal beams in our experiments. Different beams are generated by rotation of the mirror RM. Let $I_{r}$ and $I_{s}$ represent the intensities of the reference and signal beams, respectively. The diffraction efficiency of the,recorded holograms is monitored by illuminating the crystal with the phase-conjugated reference beam, $R 1^{*}$. The path of the diffracted readout beam to the output charge-coupled device (CCD) is shown as a dashed line in the figure. A self-pumped $\mathrm{BaTiO}_{3}$ phase-conjugate mirror is used to generate the conjugate wave. In addition to providing automatic alignment of the conjugate beam, the phase-conjugate mirror compensates for phase distortions due to imperfections in the SBN. When the diffraction efficiency of the holograms becomes unacceptably low, the recorded holograms are copied from the SBN into a second holographic medium, which in our experiments is a thermoplastic plate. The thermoplastic hologram is formed by using the light diffracted by the SBN hologram and reference wave R2. Shutters S2 and S4 are closed. The hologram written on the thermoplastic plate is copied back to the SBN by reference beams R1 and $\mathrm{R}^{*}$ (see Fig. 1). The intensity of $\mathrm{R}^{*}$ is selected to make the intensity of the signal beam diffracted from the thermoplastic equal to the original signal intensity, $I_{s}$. Shutters S1 and S5 are closed during this step. The result is a rejuvenated hologram of

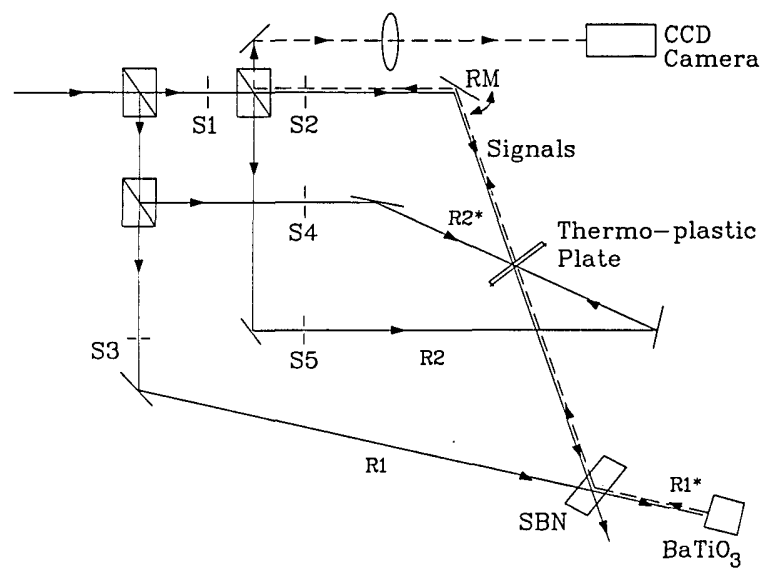

Fig. 1. Experimental system. 
each of the signal beams in the SBN. At this point, we begin adding a new series of holograms to those already stored in the SBN. When the diffraction efficiency of the holograms again falls unacceptably low, we repeat the copying process.

The temporal behavior of photorefractive holograms may be described by growth in the amplitude of the index perturbation proportional to $\left(1-e^{-\alpha I t}\right)$ during recording and decay proportional to $e^{-\alpha I t}$ during the recording of successive holograms. ${ }^{8,9} I$ is the spatially averaged recording intensity, and $\alpha$ is a constant determined by materials parameters and the recording geometry. The amplitude of the perturbation corresponding to the $m$ th hologram when $M$ holograms are recorded is

$$
A_{m}=A_{0}\left[1-\exp \left(-\alpha I t_{m}\right)\right] \exp \left(-\sum_{m^{\prime}=m+1}^{M} \alpha I t_{m^{\prime}}\right),
$$

where $A_{0}$ is the saturation amplitude and $t_{m}$ is the recording time of the $m$ th hologram. The perturbation created in the photorefractive hologram is

$$
\Phi(\mathbf{r})=\sum_{m} A_{m} f_{m}(\mathbf{r})
$$

where $f_{m}(\mathbf{r})$ describes the normalized spatial structure of the $m$ th hologram. Requiring that $A_{m}$ be a constant for all $m$ results in the following exposure schedule:

$$
t_{m}=(\alpha)^{-1} \ln \left[\frac{1+(m-1) \chi}{1+(m-2) \chi}\right],
$$

where $\chi=1-\exp \left(-t_{1} \alpha I\right)$. The schedule described in Ref. 1 corresponds to Eq. (3) for the case $\chi=1$ and ( $t_{1}$ $\rightarrow \infty$ ). We have recorded and reconstructed as many as 111 holograms in SBN by using this schedule without copying. The uniformity of the holograms was good, but, as expected, the diffraction efficiency per hologram was less than $10^{-4}$ times the saturation diffraction efficiency for a single hologram.

We begin the experiment by recording a series of holograms in the system of Fig. 1 with $M_{1}$ exposures on the SBN following the schedule of Eq. (3) for $\chi=1$. When $t_{M_{1}}$ is substituted from Eq. (3) into Eq. (1), the amplitude of the space charge for each hologram is found to be $A_{m}=A_{0} / M_{1}$ for all $m$. If $M_{1}$ is large enough, then the diffraction efficiency per hologram is small, and the diffraction efficiency of the $m$ th hologram, $\eta_{m}$, is proportional to $\left|A_{m}\right|^{2}$. This implies that $\eta_{m}=\eta_{0} / M_{1}^{2}$, where $\eta_{0}$ is proportional to $\left|A_{0}\right|^{2}$. After $M_{1}$ exposures, we copy the holograms summed in the SBN onto the thermoplastic. We then copy back to the SBN as described above. The amplitude of the saturation space charge in a photorefractive hologram is proportional to the modulation depth with which it is recorded. Since the intensity of the signal beam reconstructed from the thermoplastic hologram is equal to $I_{s}$, the intensity of each of the $M_{1}$ equally recorded components is $I_{s} / M_{1}$. The modulation depth for each component is the ratio of $\left(I_{r} I_{s} / M_{1}\right)^{1 / 2}$ to the total recording intensity $\left(I_{r}+I_{s}\right)$. Since this modulation depth is $\bar{M}_{1}$ times less than the modulation depth for one hologram, the amplitude of the spacecharge grating for each component is $A_{m}=A_{0} / \sqrt{M}_{1}$. $\eta_{m}$ is thus $\eta_{0} / M_{1}$. The total diffraction efficiency, $\eta_{T}=$ $M_{1} \eta_{m}$, is restored to the saturation value of $\eta_{0}$.

We now begin recording a new series of holograms in the SBN. The first hologram of each new series is written until its amplitude is equal to the partially erased amplitude of the copied holograms. Let $A_{m}{ }^{(j)}$ and $t_{j m}$ be the amplitude and the exposure time of the $m$ th hologram recorded in the SBN in the $j$ th recording cycle, respectively. We would like $t_{j m}$ to be such that $A_{m}{ }^{(j)}$ is constant for all $j$ and $m$. Assuming that this is the case, we use an argument similar to that described in the previous paragraph to conclude that $A_{m}{ }^{(j)}=A_{0} /\left(\sum_{l=1}^{j-1} M_{l}\right)^{1 / 2}$ at the start of the $j$ th recording cycle, i.e., just after the hologram is copied for the $(j-$ 1)th time. $M_{j}$ is the number of exposures that we make in the jth cycle. The perturbation in the crystal at the start of the $j$ th recording cycle is

$$
\begin{aligned}
\Phi\left(\mathbf{r}^{\prime}\right) & =\sum_{l=1}^{j-1} \sum_{m=1}^{M_{l}} A_{m}{ }^{(l)} f_{m}{ }^{(l)}\left(\mathbf{r}^{\prime}\right) \\
& =\frac{A_{0}}{\left(\sum_{l=1}^{j-1} M_{l}\right)^{1 / 2}} \sum_{l=1}^{j-1} \sum_{m=1}^{M_{l}} f_{m}{ }^{(l)}\left(\mathbf{r}^{\prime}\right) .
\end{aligned}
$$

The amplitude of the first hologram is equal to the partially erased amplitude of the copied holograms if

$$
A_{0}\left[1-\exp \left(-t_{j 1} \alpha I\right)\right]=\frac{A_{0}}{\left(\sum_{l=1}^{j-1} M_{l}\right)^{1 / 2}} \exp \left(-t_{j 1} \alpha I\right) .
$$

Let $\chi^{(j)}=1-\exp \left(-t_{j 1} \alpha I\right)$; Eq. (5) yields $\chi^{(j)}=1-$ $\left[\left(\sum_{l=1}^{j-1} M_{l}\right)^{1 / 2} / 1+\left(\sum_{l=2}^{j-1} M_{l}\right)^{1 / 2}\right]$. Letting $\chi=\chi^{(j)}$ in the exposure schedule of Eq. (3), we make $M_{j}$ exposures in the $j$ th cycle to record $\sum_{l=1}^{j} M_{l}$ holograms with equal amplitudes. In order to maintain a constant diffraction efficiency from the thermoplastic, we select $M_{j}$ such that the total diffraction efficiency of the summed hologram on the SBN falls back to its value after the first $M_{1}$ exposures, i.e., $\eta_{0} / M_{1}$. Each time $M$ $=\sum M_{i}$ holograms are copied back and forth, the diffraction efficiency for each hologram is restored to $1 / M$.

Figure 2 is a log-log plot of the mean diffractiondiffraction efficiency per hologram relative to the diffraction efficiency for a single exposure versus the number of exposures. The figure shows experimental results for recording as many as 25 holograms in a $\mathrm{SBN}$ :Ce crystal. The diffracted phase-conjugate reference for each hologram was monitored by the CCD shown in Fig. 1. The solid curve in Fig. 2 corresponds to the theoretical $M^{-2}$ decay in the diffraction efficiency per hologram when Eq. (2) is followed with $\chi=1$. The *'s are experimental data points for the mean diffracted power of holograms stored according to this schedule. The dashed curve shows the theoretical path followed by the diffraction efficiency per hologram with periodic copying when $M_{1}=5$. The \#'s show experimental data points found when this approach was followed. The dotted curve corresponds to a decrease in diffraction efficiency proportional to 


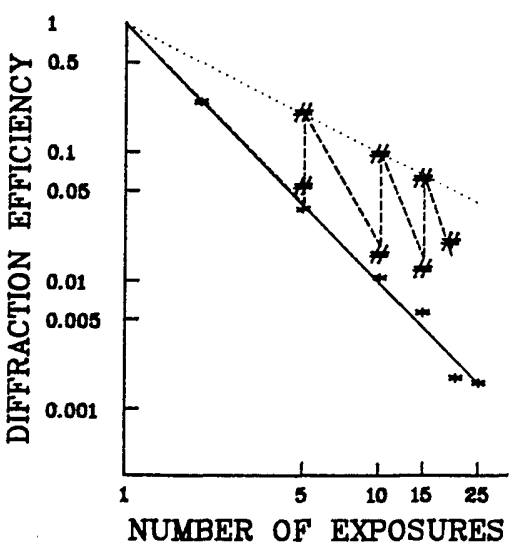

Fig. 2. Relative diffraction efficiency of each hologram versus number of exposures (log-log scale).

$M^{-1}$. Thermoplastic holograms were made after 5,10 , and 15 exposures.

One might like to implement periodically refreshed storage by using two photorefractive media. Unfortunately, copying between two photorefractive crystals is complicated by the decay of the hologram being copied while the second hologram is recorded. Suppose that we wish to copy a hologram between two identical photorefractive crystals. As we read out the first hologram, the light intensity diffracted from it decays exponentially at a rate proportional to the readout intensity. The rate at which the second hologram builds up is determined by the overall intensity incident upon it. If the reference beam for the second hologram is too bright, then the modulation depth is too weak; if it is too low, then the second hologram does not build up sufficiently before the first hologram decays. If we set the intensity of the reference beam such that the modulation depth is constant at the second hologram, then the overall diffraction efficiency of the copied hologram can exceed the efficiency of the original only if the strength of the perturbation produced in the crystal by a single exposure exceeds the strength required to achieve (in principle) $100 \%$ diffraction efficiency. This can be accomplished by selecting a crystal with the appropriate combination of electro-optic coefficient, dielectric constant, index, and thickness. For example, the critical thickness for holograms using the $r_{51}$ electro-optic coefficient in barium titanate is approximately $2 \mathrm{~mm}$. Since crystals of such thickness are readily available, it should be possible to extend the copying technique to an allphotorefractive system.

We have described periodic copying between two holograms, using two key simplifications: The holographic media are planar, which implies that they can be copied in one exposure, and there is a fixed number of exposures $M$ that need to be done. The implementation of learning algorithms in optical neural networks ${ }^{1}$ is an important class of problems that requires an arbitrary number of exposures. From Eq. (4) we see that the perturbation that accumulates in the crystal is simply the average of all the exposures, independently of their number. If the individual exposures are statistically independent, then the sum in Eq. (4) will grow in proportion to $\sqrt{M}$. This will precisely counterbalance the $M^{1 / 2}$ factor in Eq. (4), yielding a steady-state diffraction efficiency that is independent of $M$. If the individual exposures are correlated (a case that we have not analyzed in this Letter), then the sum can grow faster than $\sqrt{M}$. In this case the reduction in diffraction efficiency due to an additional exposure would have been less that what we calculated. The copying process recovers this smaller loss in diffraction efficiency, and the steady-state overall efficiency remains unchanged and independent of $M$.

The extension of periodically refreshed recording to holograms utilizing volume degrees of freedom is complicated by the fact that a volume hologram cannot in general be evaluated or copied in a single exposure. ${ }^{10,11}$ We define the rank $R$ of a hologram to be the minimum number of exposures needed to form the hologram. If the complete hologram is not known $a$ priori, i.e., if we wish to record holograms adaptively, it is not possible to ensure that the minimal number of exposures will be used in recording. The copying technique described here allows us to recover the loss in diffraction efficiency that is due to exposing a hologram more than $R$ times. In our experiments $R$ was limited to 1 by our use of a single reference beam. For holograms of higher rank, $R$ references would be required. Using appropriate reference signals to copy a stored hologram results in an increase in the diffraction efficiency of each component of the hologram by the factor $M$ / $R^{2}$, compared with the efficiency obtained without copying, where $M$ is the number of exposures used to form the original hologram. In the simplest case the steady-state efficiency is again independent of $M$.

This research is supported by the Defense Advanced Research Projects Agency. The authors thank Rockwell International and Ratnakar Neurgaonkar for the SBN used in these experiments. David Brady acknowledges the support of the Aerojet Corporation through the Caltech Program for Advanced Technology.

\section{References}

1. D. Psaltis, D. Brady, and K. Wagner, Appl. Opt. 27, 1752 (1988).

2. K. Blфtekjaer, Appl. Opt. 18, 57 (1979).

3. W. J. Burke and P. Sheng, J. Appl. Phys. 48, 681 (1977).

4. J. C. Palais and J. A. Wise, Appl. Opt. 10, 667 (1971).

5. K. M. Johnson, M. Armstrong, L. Hesselink, and J. W. Goodman, Appl. Opt. 24, 4467 (1985).

6. D. Psaltis, D. Brady, X.-G. Gu, and K. Hsu, in Optical Processing and Computing, H. Arsenault, ed. (Academic, Orlando, Fla., 1989).

7. H. Lee, X. G. Gu, and D. Psaltis, J. Appl. Phys. 65, 2191 (1989).

8. N. V. Kuktarev, V. B. Markov, S. G. Odulov, M. S. Soskin, and V. L. Vinetskii, Ferroelectrics 22, 949 (1979).

9. T. J. Hall, R. Jaura, L. M. Connors, and P. D. Foote, Prog. Quantum Electron. 10, 77 (1985).

10. E. Wolf, Opt. Commun. 1, 153 (1969).

11. S. Hudson, D. J. Brady, and D. Psaltis, in Digest of Optical Society of America Annual Meeting (Optical Society of America, Washington, D.C., 1988), p. 74. 